\title{
Magnetic Relaxation in Coaxial Helicity Injection Discharges in the HIT-II Spherical Torus
}

\author{
Aaron J. Redd* \\ Will T. Hamp* \\ Non-member \\ Thomas R. Jarboe* \\ Non-member \\ Brian A. Nelson* \\ Non-member \\ R. Griff O'Neill* \\ Non-member \\ Roger J. Smith* \\ Non-member \\ Non-member
}

\begin{abstract}
Recent Coaxial Helicity Injection (CHI) studies using the Helicity Injected Torus device (HIT-II) have produced discharges with toroidal plasma currents up to $350 \mathrm{kA}$. Direct measurements using an internal magnetic probe array show a total poloidal flux in the confined plasma significantly greater than the vacuum injector flux, confirming both the unambiguous presence of a closed-flux core region and the generation of poloidal flux through magnetic relaxation. The key innovation for producing these discharges is a sufficiently high ratio of CHI injector current to toroidal field current, quantified as the dimensionless product $\lambda_{\mathrm{INJ}} d$ greater than 0.3 , where $d$ is the effective distance between the electrodes and $\lambda_{\text {INJ }}$ is the inverse magnetic scale length associated with the CHI injector (defined as $\mu_{0} I_{\mathrm{INJ}} / \psi_{\mathrm{INJ}}$, where $I_{\mathrm{INJ}}$ is the injector current and $\psi_{\text {INJ }}$ is the poloidal injector flux connecting the electrodes). This critical value of $\lambda_{\text {INJ }} d$ is understood as a balance between two competing processes: reconnection in the injector generating closed poloidal flux, and resistive decay of the closed flux and current. A value of $\lambda_{\mathrm{INJ}} d>0.3$ corresponds to a rate of relaxation greater than the rate of resistive decay, allowing the build-up of poloidal flux and toroidal current.
\end{abstract}

Keywords: magnetized plasma, spherical torus, helicity injection

\section{Introduction and the HIT-II Device}

The Helicity Injected Torus (HIT) program is dedicated to the development of helicity injection current drive in magnetically-confined toroidal plasmas ${ }^{(1)}$. The HIT-II device is a spherical torus or $\mathrm{ST}^{(2)(3)}$ capable of forming and sustaining tokamak discharges using either Coaxial Helicity Injection (CHI) or Ohmic drive. Previous HIT-II CHI studies have exclusively used high toroidal fields (total central column current $I_{\mathrm{TF}} \approx 800$ $\mathrm{kA}$ ), and have generated tokamak plasmas with toroidal plasma currents $I_{p}$ up to $200 \mathrm{kA}$ consistently, some discharges transiently as high as $240 \mathrm{kA}$. The HIT-II CHI studies described here involve a more optimum operating regime, in which $I_{p}$ can consistently exceed 300 $\mathrm{kA}$, and transiently reach or exceed $350 \mathrm{kA}$. The CHI discharges in this new operating regime can easily be distinguished from discharges in previous studies. After the rapid "bubble-burst" and discharge formation, the plasma current, electron density, radiated power and poloidal flux in these new discharges all continue to increase with time. This slow post-formation buildup distinguishes these discharges from the transient-CHI formation studies reported by Raman ${ }^{(4)}$ and Nagata ${ }^{(10)}$.

\footnotetext{
* Aerospace and Energetics Research, University of Washington Box 352250, Seattle, Washington 98155-2250 USA
}

Internal magnetic probing in these current-buildup discharges shows that the poloidal flux reaches an amount many times the injector flux, and indeed significantly higher than the sum of the open vacuum fluxes, providing experimental verification of poloidal flux generation. Note, for all discharges described here, there is no inductive current drive applied, only CHI.

The HIT-II device, shown in cross-section in Fig. 1, has a real-time flux feedback control system and an extensive diagnostic set, which have been described in detail in Refs. ${ }^{(1)}$ and ${ }^{(2)}$. Some of the HIT-II magnetic and spectroscopic diagnostic systems are especially important for describing the data presented in this manuscript, and so will be discussed briefly. Poloidal magnetic fluxes are measured by a set of 24 flux loops on the outer surface of the HIT-II vacuum vessel, with each flux loop typically quite close to one of the poloidal field coils. These flux loops are used by the boundary flux feedback system, with the measured flux compared to a preprogrammed demand, and any difference fed back as a change in the current in the nearby poloidal field coil. Magnetic fields at the plasma edge are measured with two poloidal arrays of triple magnetic field probes mounted in the plasma-facing surface of the HIT-II shell. Poloidal currents in the outboard shell are measured at the HIT-II midplane by two toroidal arrays of Rogowski coils, which measure this poloidal current as 


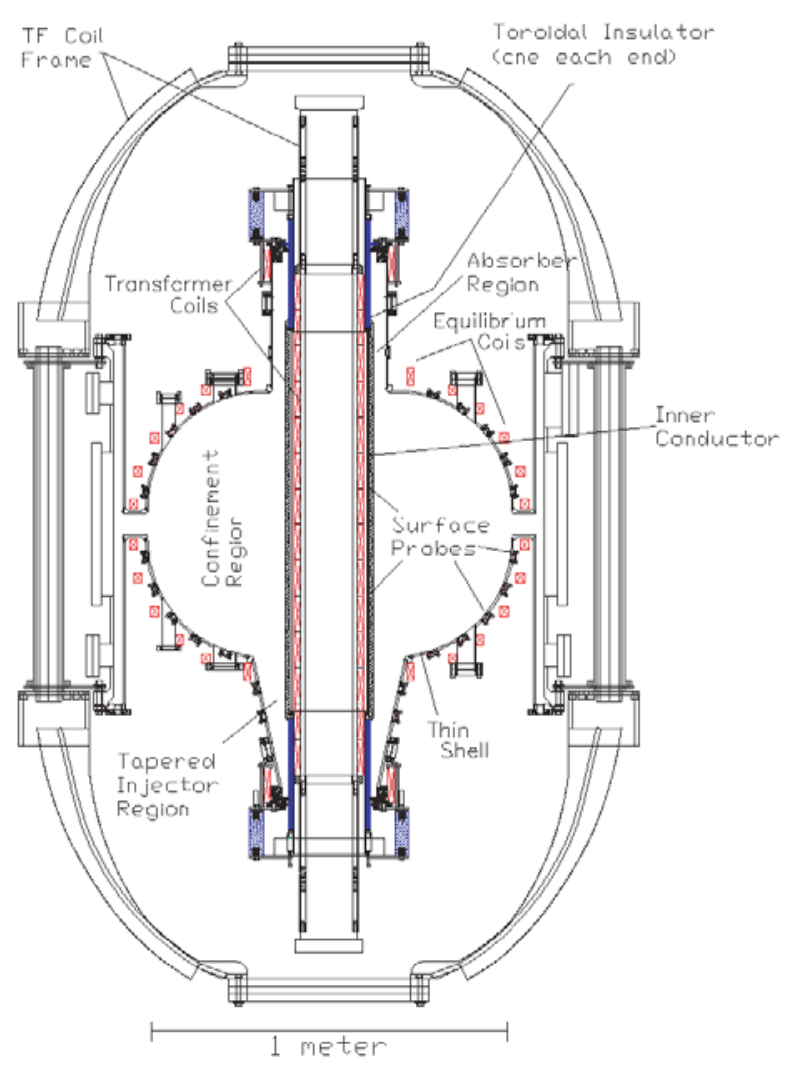

Fig. 1. Schematic layout of the HIT-II device; The location and shape of each of the 28 Poloidal Field $(\mathrm{PF})$ coils is marked with in red, and the toroidal insulating breaks indicated in blue. Near each PF coil is a corresponding flux loop, used in the flux feedback system. Also indicated are some of the 34 poloidal locations of surface-mounted magnetic triple probes

the difference between the toroidal fields inside and outside the outer shell of the device. The poloidal wall current is independently measured by the poloidal arrays of surface-mounted magnetic triple probes, which can also indicate where on the shell the injector current leaves the wall and enters the plasma. These two measurements agree closely for the poloidal wall current at the HIT-II midplane, both for calibration pulses and plasma pulses. The internal probing array consists of a single probe "stem", containing an array of eight triple magnetic field probes, spaced approximately $12 \mathrm{~mm}$ apart, with a boron nitride outer sheath. The ion Doppler spectrometer (IDS) is a single-chord high-resolution diagnostic, tunable to frequencies ranging from near-UV up through the visible range (spacings between the 16 wavelength channels are less than $0.025 \mathrm{~nm}$ ). By tuning the IDS to a particular impurity-ion emission, the spectrometer can be used to measure the chord-averaged ion temperature and flow velocity, as described in Ref. ${ }^{(5)}$.

For the discharges described here, the boundary fluxes form an unbalanced Double-Null Divertor (DND) configuration. That is, the lower divertor ("injector") has more poloidal flux connecting the electrode surfaces than the upper divertor ("absorber"), with this difference defined as the injector flux $\psi_{\text {INJ }}$. The boundary flux configuration is called "HIT-like", as it is similar to the vacuum flux configuration of the HIT device ${ }^{(1)(6)}$. In the injector region, the boundary flux intersects the central column between the third and fourth coils (shown in red in Fig. 1) and intersects the outer shell along the length of the tapered cone (between the two widely spaced coils at the ends of the cone). In the absorber region, the boundary flux is drawn across the short gap from the central column to the right-angle lip of the confinement region. Vacuum magnetic field calculations show that these boundary fluxes do not extend significantly into the confinement region, instead remaining for the most part betwen the electrodes in the injector and absorber regions. Before each discharge, plasma sources located in the injector region inject ionized deuterium near the poloidal injector flux ${ }^{(7)}$. Neutral deuterium gas is also puffed at either or both electrode surfaces. Each discharge begins by applying a high-voltage lowcapacitance formation bank to the electrodes; this bank current, flowing radially along the injector flux, is subject to a $J \times B$ force due to the externally-generated toroidal field, "injecting" the plasma (with embedded injector flux and injector current) into the confinement region. As the formation bank is exhausted (typically after 200 to 400 microseconds), "rows" of the lowervoltage, higher-capacitance sustainment bank are fired in a prescribed sequence, singly or in sets. The total bank current $I_{\mathrm{BANK}}$ is the sum of the formation and sustainment bank currents. In many CHI discharges, a significant fraction of $I_{\mathrm{BANK}}$ does not participate in the main discharge in the confinement region, instead travelling poloidally through the HIT-II shell to the coaxial absorber region and then conducted from the shell to the central column by a glow plasma at the absorber. The toroidal array of gap Rogowskis and the poloidal array of surface-mounted magnetic field probes can be used to independently measure this wall current $I_{\mathrm{WALL}}$, and also to demonstrate that all of $I_{\mathrm{WALL}}$ leaves the shell at the absorber. The magnitude of $I_{\text {WALL }}$ is loosely related to wall conditioning and the flux boundaries (fresh titanium gettering and the maintenance of a poloidal field null in the absorber region both reduce $\left.I_{\text {WALL }}\right)$. Throughout this manuscript, the CHI injector current is defined as $I_{\text {INJ }}=I_{\mathrm{BANK}}-I_{\mathrm{WALL}}$. Wall conditioning in these studies is achieved by frequent titanium getting, with the HIT-II base pressure consistently less than $10^{-8}$ Torr.

The key to forming and sustaining discharges in this new operating regime is increasing the poloidal injector flux relative to the externally-generated toroidal field, either by reducing the toroidal field current $I_{\mathrm{TF}}$ or increasing the injector flux $\psi_{\text {INJ }}$. Either change has the tendency to increase the injector current $I_{\mathrm{INJ}}$, but this operating regime in HIT-II can still be accessed with relatively modest bank currents (less than $40 \mathrm{kA}$ throughout the discharge, as shown in Fig. 3). Previous HITII CHI studies were performed with an imposed engineering constraint that $I_{\mathrm{BANK}}$ would not exceed $30 \mathrm{kA}$, which may be too small to consistently produce $\mathrm{CHI}$ discharges that build up toroidal current and poloidal 
flux. This engineering constraint was lifted for the final HIT-II experimental campaign, leading to discovery of this more optimum operating regime.

\section{Experimental Results}

The key plasma parameters versus time for HIT-II discharge \#30386 are shown in Figure 2. Note that this discharge has the highest measured toroidal plasma current ever measured in HIT-II, with a peak value of 353 $\mathrm{kA}$. The absolute magnitude of the injector current in this discharge is unknown within $10 \mathrm{kA}$, but the overall time evolution of $I_{\text {INJ }}$ is accurate (for this particular discharge, the sustainment bank current measurement is affected by a transient short in the Rogowski coil measuring $\left.I_{\mathrm{BANK}}\right)$. Interferometry in these high-performance CHI discharges typically shows a large-magnitude density transient during the initial rapid current rise, a settling down to a lower level, then a subsequent slower density rise to a peak coinciding with the maximum current, followed by a final decay period. In absolute terms, the average electron density in these $\mathrm{CHI}$ discharges is approximately $3 \times 10^{19} \mathrm{~m}^{-3}$ at the start of the slow rise, and peaks at approximately $10 \times 10^{19} \mathrm{~m}^{-3}$ (both densities are estimated to be correct to within a factor of two). This time history of the electron density corresponds to the evolution of the total radiated power, shown in Fig. $2(\mathrm{~d})$. The toroidal field current $I_{\mathrm{TF}}$ for discharge \#30386 is approximately $450 \mathrm{kA}$.

The key plasma parameters versus time for HIT-II discharge \#29133 are shown in Figure 3. Aside from some transients before and after the rapid initial plasma current rise, the injector current $I_{\mathrm{INJ}}$ is approximately

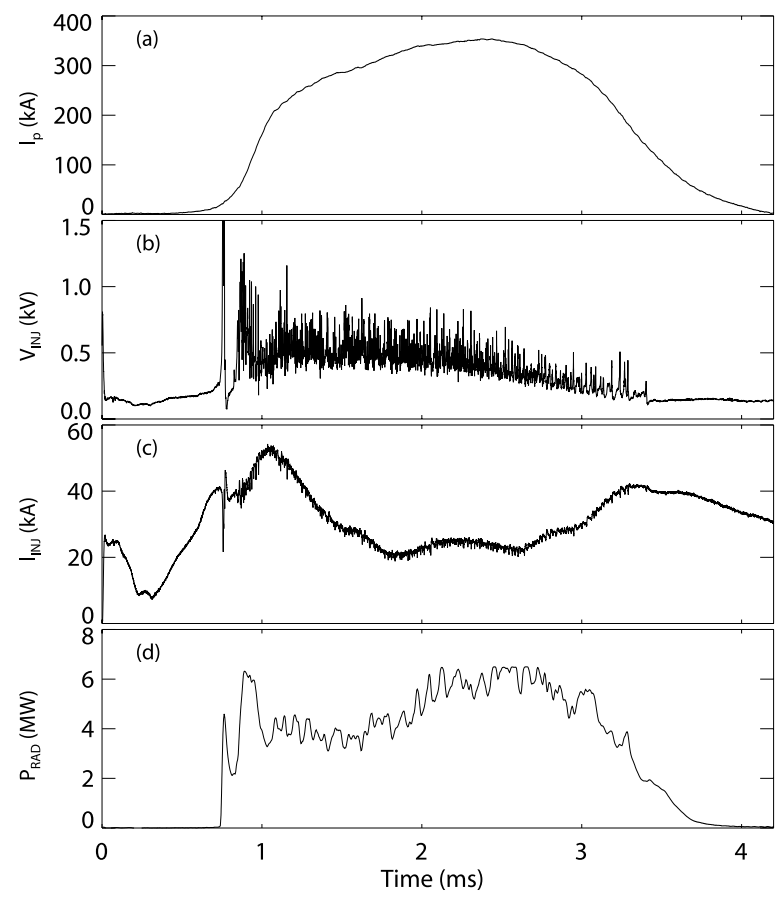

Fig. 2. (a) Toroidal plasma current, (b) injector voltage, (c) injector current $I_{\mathrm{INJ}}$, and (d) total radiated power from the confinement region of HIT-II versus time for double-null low-TF CHI discharge \#30386 constant throughout the rest of the discharge, as expected $^{(3)}$. The plasma current $I_{p}$ and poloidal wall current $I_{\text {WALL }}$ rise until $3.3 \mathrm{~ms}$, approximately coinciding with the exhaustion of the sustainment bank and a steady drop in the injector voltage $\left(I_{\mathrm{TF}}\right.$ for discharge \#29133 is approximately $470 \mathrm{kA}$ ).

Probing studies of these low-TF discharges have found these plasmas can tolerate shallow probing (probe array inserted radially inward up to $90 \mathrm{~mm}$ from the wall at the midplane gap), with only slight degradation to the plasma current $I_{p}$. Figure 4 shows $I_{p}$ and calculated poloidal flux (relative to the plasma-facing wall, assuming axisymmetry) for HIT-II discharge \#29388, with the probe array inserted $90 \mathrm{~mm}$ (innermost field probe at $R=0.42 \mathrm{~m}$, outermost field probe at $R=0.51$ $\mathrm{m}$, and the six probes in between spaced evenly $12 \mathrm{~mm}$ apart). The seven traces shown in part (b) are the poloidal fluxes measured by each probe, relative to the outermost probe, which is located approximately at the plasma-facing surface. In part (c), only the flux for the innermost probe is shown, with horizontal dashed lines corresponding to $6 \mathrm{mWb}$ (equal to the programmed and measured injector flux $\left.\psi_{\text {INJ }}\right)$ and $11 \mathrm{mWb}$. The peak measured poloidal flux at the innermost probe is approximately $17 \mathrm{mWb}$, relative to the outer wall ( $I_{\mathrm{TF}}$ for discharge \#29388 is approximately $320 \mathrm{kA}$ ). This peak measured flux is a robust measurement, in the sense that the peak measured flux in each of five repeat pulses (repeats of \#29388) is approximately $17 \mathrm{mWb}$. Deeper probing, placing the probe tips at a depth of $155 \mathrm{~mm}$, puts the outermost magnetic field probe at the location of the inntermost probe in shallow probing $(R=0.42 \mathrm{~m})$. Deep probing significantly degrades the

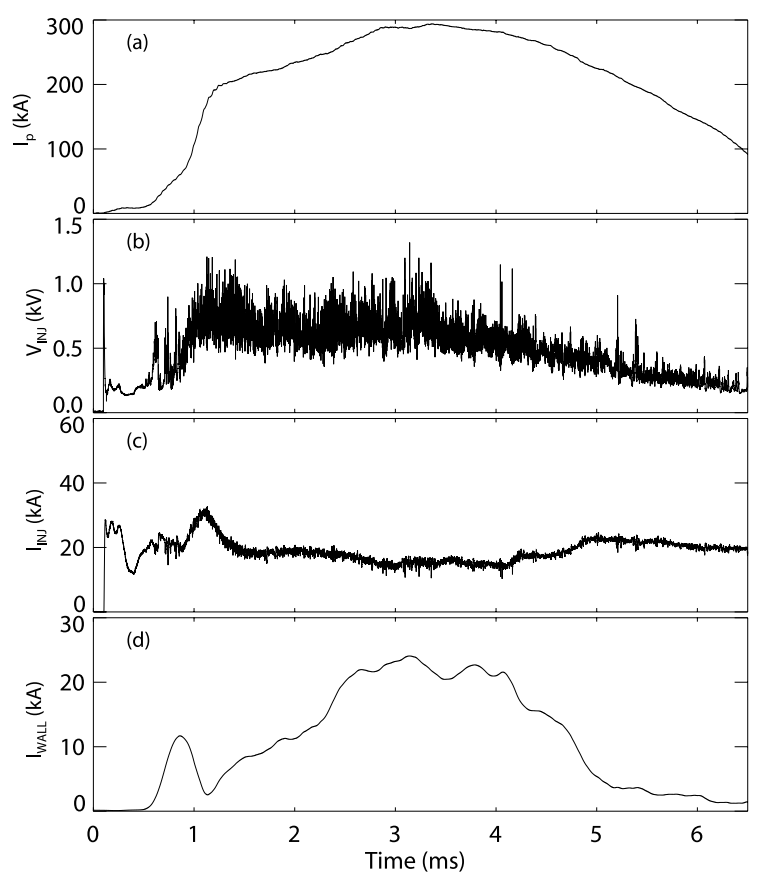

Fig. 3. (a) Toroidal plasma current, (b) injector voltage, (c) injector current, and (d) poloidal wall current versus time for HIT-II double-null low-TF CHI discharge \#29133 


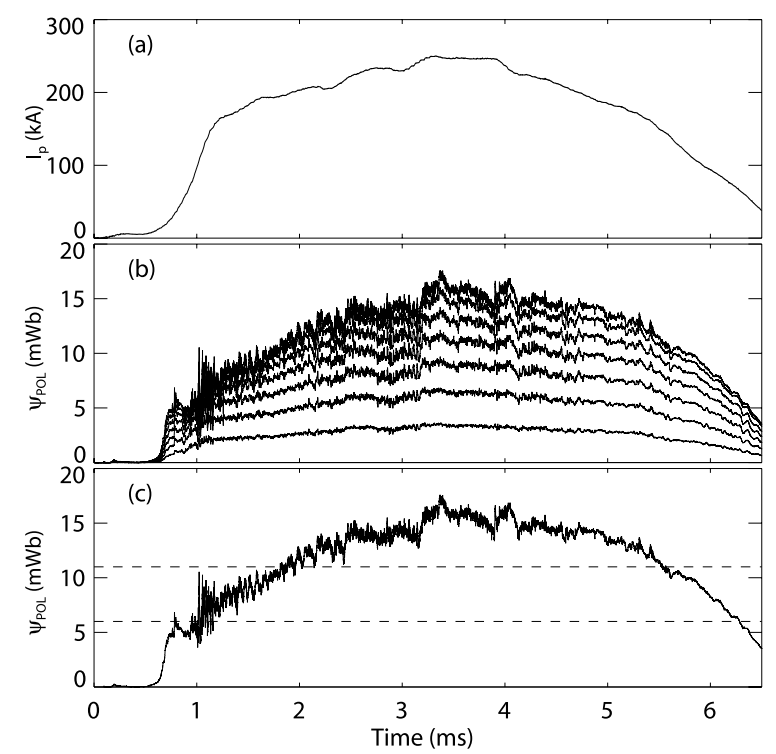

Fig. 4. Versus time in HIT-II double-null low-TF CHI discharge \#29388, (a) toroidal plasma current, (b) probe-measured poloidal flux for the seven inner probes of the internal array, relative to the wall, and (c) probe-measured poloidal flux for only the innermost probe (located at $R=0.42 \mathrm{~m}$ ). The dashed lines in (c) indicate $6 \mathrm{mWb}$ and $11 \mathrm{mWb}$

overall plasma performance (peak $I_{p}$ reduced from approximately $250 \mathrm{kA}$ to approximately $200 \mathrm{kA}$ ), but the probe spans the magnetic axis, which is located between $R=0.35 \mathrm{~m}$ and $R=0.40 \mathrm{~m}$ throughout the duration of these deeply-probed discharges. Assuming axisymmetry, there is an additional 1 to $2 \mathrm{mWb}$ of poloidal flux between $R=0.42 \mathrm{~m}$ and the magnetic axis in the deeply probed (and slightly degraded) discharges.

Note, the measured poloidal fields vary significantly from probe location to probe location at each time during the discharge, especially after $t=2 \mathrm{~ms}$. Assuming axisymmetry, this deviation in the poloidal fields along the length of the probe indicates that the probe is intersecting significant toroidal current all along its length. Similarly, the current-carrying region of the plasma extends almost all the way out to the plasma-facing surface of HIT-II, at the outermost probe location of $R=0.51 \mathrm{~m}$. Expressed differently, the internal probe data is consistent with a non-current-carrying region at the edge of discharge \#29388, but this region is no wider than the interval from the outermost field probe to its nearestneighbor field probe in the array $(12 \mathrm{~mm})$, and therefore the poloidal flux in this non-current-carrying region is no greater than $3 \mathrm{mWb}$.

Spectroscopic measurements of these discharges show significant line radiation for a range of ion masses and charge states, from Deuterium recombination up to Carbon-V emission. Strong edge-localized OxygenV emission (with wavelength $278 \mathrm{~nm}$ ) produced highquality reproducible ion Doppler results from sets of identical discharges. Ion temperature and toroidal flow measurements from discharge \#29141, a typical discharge in this new operating regime, are shown in Fig. 5. For this discharge, the IDS is viewing an edge chord in

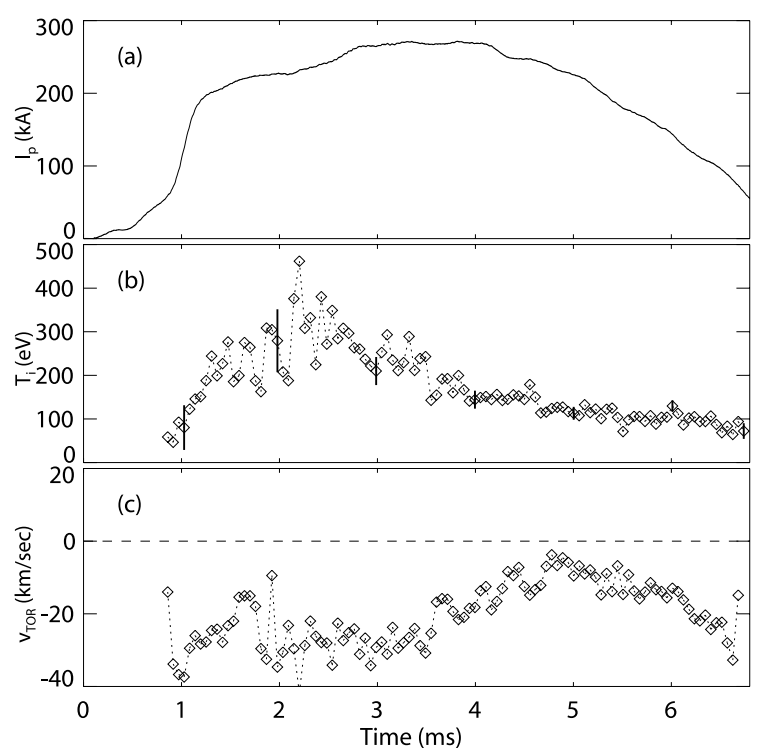

Fig. 5. (a) Toroidal plasma current, (b) average ion temperature, and (c) average ion toroidal flow velocity for an edge chord in HIT-II double-null low-TF CHI discharge \#29141; Error bars for the ion temperatures are shown for some representative points

the HIT-II midplane, a chord tangent to an impact parameter of $R=0.44 \mathrm{~m}$. The negative measured velocity indicates that the ion flow is in the direction opposite to the plasma current. Typical for these plasmas is the high ion temperature (over $200 \mathrm{eV}$ ) during the current ramp-up phase, followed by a monotonic decrease in $T_{i}$ throughout the remainder of the discharge. Calculated errors in the ion temperatures are shown for a few representative points in Fig. 5 (b). The calculated error in the toroidal flow velocity $v_{\text {TOR }}$ is not shown, but is comparable to the temporal jitter in the signal shown in Fig. $5(\mathrm{c})$.

Figure $6(\mathrm{a})$ shows the peak value of $I_{p} / I_{\mathrm{TF}}$ and Fig. $6(\mathrm{~b})$ the average post-formation current ramp-up rate, both plotted against a dimensionless measure of the magnetic field pitch in the injector for 307 HIT-II CHI discharges. The magnetic field pitch (the amount by which the direction of the field lines have changed between the surfaces of the CHI electrodes) is estimated as the inverse magnetic scale length for the injector $\lambda_{\text {INJ }}$ (defined as $\mu_{0} I_{\text {INJ }} / \psi_{\text {INJ }}$ ) multiplied by the effective inter-electrode distance $d$. The current ratio $I_{p} / I_{\mathrm{TF}}$ is related to $\lambda_{\mathrm{TOK}}$, the inverse scale length for the tokamak plasma (defined as $\mu_{0} I_{p} / \phi_{\mathrm{TF}}$, where $\phi_{\mathrm{TF}}$ is the toroidal flux in the confinement region, generated both by external coils and poloidal plasma currents). In each of these CHI discharges, a post-formation $I_{p}$ rampup continues until either the sustainment bank is exhausted or $\lambda_{\text {TOK }}$ rises to approximately equal $\lambda_{\text {INJ }}$.

Empirically, if $\lambda_{\text {INJ }} d$ is below a critical value, approximately one-third, the post-formation current rampup rate is always negligible. Although there is not a consistent exact value for this threshold for all discharges, it is possible to approach the threshold on any 


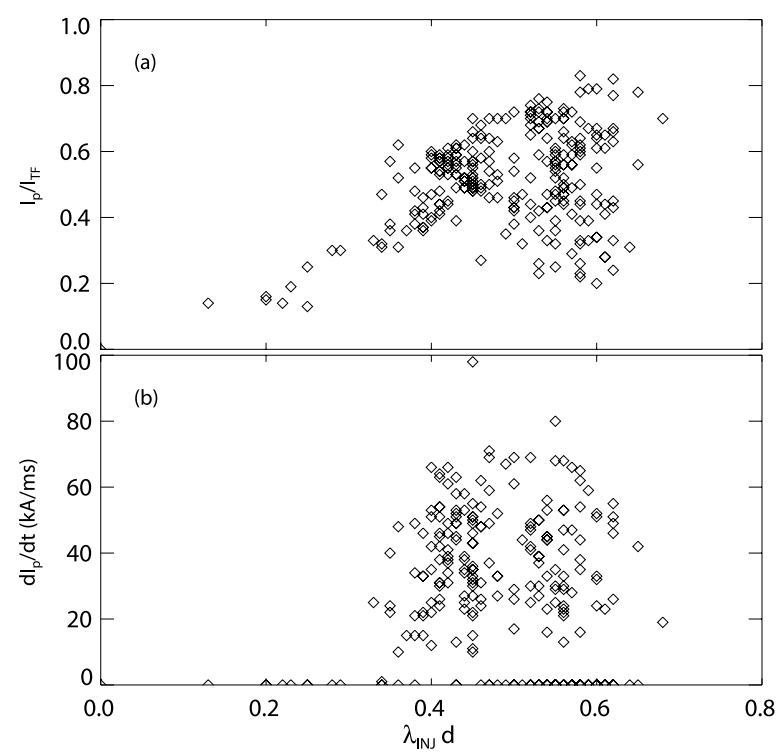

Fig. 6. For 307 double-null HIT-II CHI discharges with various toroidal field coil currents $I_{\mathrm{TF}}$, (a) the peak value of $I_{p} / I_{\mathrm{TF}}$ versus $\lambda_{\mathrm{INJ}} d$, and (b) postformation $I_{p}$ rise rate versus $\lambda_{\mathrm{INJ}} d$

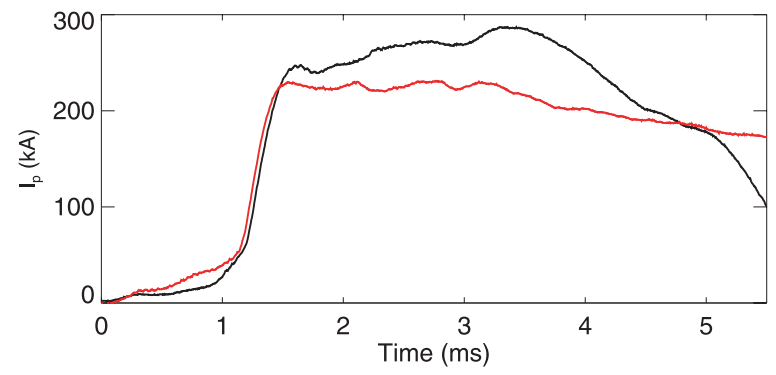

Fig. 7. Toroidal plasma currents for two HIT-II double-null high-TF CHI discharges with identical toroidal field currents: \#29473 $\left(\lambda_{\mathrm{INJ}} d=0.33\right)$ is shown in red, and \#29469 $\left(\lambda_{\mathrm{INJ}} d=0.35\right)$ in black

particular run day, and determine the "sharpness" of the build-up threshold. Figure 7 shows toroidal plasma currents for two discharges (\#29473 and \#29469) from the same run day, with nearly identical experimental parameters. The key difference between these discharges is the amount of poloidal injector flux: $\psi_{\mathrm{INJ}}=10.0 \mathrm{mWb}$ in discharge \#29473 (implying a value of 0.33 for $\lambda_{\mathrm{INJ}} d$ ), and $\psi_{\mathrm{INJ}}=11.9 \mathrm{mWb}$ for discharge \#29469 $\left(\lambda_{\mathrm{INJ}} d=\right.$ $0.35)$. Note, the post-formation plasma current is flat versus time for \#29473, but the plasma current shows a significant post-formation rise in discharge \#29469. In both of these discharges, the toroidal field current $I_{\mathrm{TF}}$ is approximately $750 \mathrm{kA}$, similar to the toroidal field currents in previous HIT-II CHI studies.

Also note, in all these discharges, the inter-electrode distance $d$ is small relative to the HIT-II minor radius.

\section{Discussion}

CHI discharges in the new HIT-II operating mode are significantly different from the CHI discharges that have previously been studied in various STs. Not least of these differences is the large post-formation ramp-up in the measured toroidal plasma current, and also the very high peak values for this current $I_{p}$. Previously, the highest plasma current observed in HIT-II was $240 \mathrm{kA}$, in a discharge quite similar to \#29473, shown in Fig. 7. The new CHI operating regime allows plasma currents to transiently reach above $350 \mathrm{kA}$ (as shown in Fig. 2) in a few discharges, and to regularly generate plasmas in which $I_{p}$ meets or exceeds $300 \mathrm{kA}$ (Fig. 3). Also noteworthy is that this post-formation current ramp occurs during a period in which the external discharge parameters (e.g., injector flux, injector current, toroidal field current) can all be held approximately constant, as in discharge \#29133 shown in Fig. 3. The increasing toroidal plasma current also correlates with increasing electron density and radiated power, which would be consistent with a build-up of plasma in the confinement region.

Two-dimensional reconstructions of ST CHI magnetic equilibria have previously indicated the presence of closed-flux plasma cores ${ }^{(2)(6)}$. Experimentally, however, the presence of such a closed-flux core has remained unproven, as these edge magnetic measurements could still be explained in terms of $I_{\text {INJ }}$ being "wrapped up" toroidally by the strong toroidal field, as described in Ref. ${ }^{(9)}$. Direct evidence for CHI generating a closedflux plasma core in this new operating mode is given by the probing data shown in Fig. 4, where the measured poloidal flux in discharge \#29388 reaches a peak value of $17 \mathrm{mWb}$, considerably larger than the injector flux of $6 \mathrm{mWb}$. Recall that this injector flux, like all poloidal flux linking the HIT-II walls, is directly measured using the set of 24 poloidal flux loops. Deeper probing of otherwise matching discharges indicates that there is an additional 1 to $2 \mathrm{mWb}$ of flux before reaching the magnetic axis, but the performance degradation in the deeply probed discharges suggests that this 1 to $2 \mathrm{mWb}$ is actually an underestimate for the additional poloidal flux in unprobed or shallowly probed discharges. Hence, the total poloidal flux in discharge \#29388, from the wall to the magnetic axis, is almost certainly in axcess of 19 $\mathrm{mWb}$. Using the notation of Nagata et al in Ref. ${ }^{(10)}$, the peak poloidal flux $\Psi_{p}$ in \#29388 is at least $19 \mathrm{mWb}$, or $\Psi_{p} \geq 19 \mathrm{mWb}$.

Detailed consideration of the flux boundary conditions in \#29388 indicates the maximum amount of open vacuum flux in this discharge that could intersect the probe is approximately $11 \mathrm{mWb}$. This total open flux includes the $6 \mathrm{mWb}$ injector flux, an additional $2.5 \mathrm{mWb}$ flux drawn across both the injector and absorber gaps (to create the second DND X-point at the absorber), and the remainder is vertical field. During a discharge, the additional flux across the injector and absorber gaps is assumed to reconnect away, leaving an additional 2.5 $\mathrm{mWb}$ of vertical field on the inboard and outboard sides of the plasma. Recall, all boundary-intersecting fluxes are measured by the flux loops, and the flux feedback system will prevent the magnetic equilibrium from deviating significantly from these prescribed boundary flux demands. Assuming a worst-case scenario, where all of this open poloidal flux is intersecting the probe, and 
not pushed into the stainless-steel wall behind the last probe windings at $R=0.51 \mathrm{~m}$, the open flux $\Psi_{\text {po }}=11$ $\mathrm{mWb}$ and the corresponding closed flux $\Psi_{\mathrm{pc}}$ is at least $8 \mathrm{mWb}$. Compared to the measured bias flux (injector flux) of $6 \mathrm{mWb}$, the worst-case values for the ratio $M_{\Psi}$ and poloidal flux amplification ratio $A_{\Psi}$ in \#29388 are 0.31 and 1.3 , respectively. However, recall that the internal probing measurements of \#29388 indicate that the current-carrying flux is very close to the plasma-facing wall, with no more than $3 \mathrm{mWb}$ of non-current-carrying poloidal flux intersecting the entire length of the probe. Consistency with the probing data requires either: the second DND X-point did not form, despite having two sheets of opposing poloidal flux pushed together near the absorber; or, a significant amount of the vertical field, which connects the outer shell to itself, is carrying current. Neither of these scenarios is physically plausible, which suggests that the original assumption (all open flux is intersecting the probe) is flawed. This suggestion is supported by previous equilibrium reconstructions of HIT-II CHI discharges in Refs. ${ }^{(2)(8)}$, in which the poloidal flux associated with vertical field is pushed out of the confinement region and into the stainless-steel shell. As noted above, if the vertical field flux in discharge \#29388 is pushed into the HIT-II shell, then it is located behind the probing array, and should not be included in an estimate of $\Psi_{\text {po }}$. With these physical effects in mind, the open flux in discharge \#29388 as measured by the internal probe is estimated to be no more than the prescribed injector flux of $6 \mathrm{mWb}$, or $\Psi_{\text {po }} \leq 6 \mathrm{mWb}$. The corresponding closed flux is at least $13 \mathrm{mWb}\left(\Psi_{\mathrm{pc}} \geq 13 \mathrm{mWb}\right)$, which yields $A_{\Psi} \geq 0.68$ and $A_{\Psi} \geq 3.2$. If a substantial amount of the bias flux remained in the injector region (as seen in equilibrium reconstructions for discharges in the HIT and HIT-II devices ${ }^{(1)(2)(6)(11)}$ ), then the corresponding reduction in the estimated $\Psi_{\text {po }}$ would increase the poloidal flux amplification factor $A_{\Psi}$ even more. In any case, the calculated values of $M_{\Psi}$ and $A_{\Psi}$ above are reasonable compared to the plasma performance described in Fig. 5 of Ref. ${ }^{(10)}$, especially for the so-called "tokamak regime" (for which the observed values of $M_{\Psi}$ and $A_{\Psi}$ in the Helicity Injected Spherical Torus device were approximately 0.45 and 3.0, respectively). Finally, note that discharge \#29388 is not an optimum CHI configuration, but is a plasma convenient for probing studies; there are many CHI discharges with significantly higher peak plasma currents (especially \#30386 and \#29133, in Figs. 2 and 3, respectively), which could be expected to exhibit more poloidal flux if probed.

This interpretation of the poloidal flux amplification in discharge \#29388 is further supported by the temporal behavior of the poloidal flux measurements in Fig. 4. As the current $I_{p}$ rises rapidly during the plasma formation, the measured poloidal fluxes rapidly rise, but then level off or even drop slightly before rising again, as might be expected if a significant amount of reconnection were occurring at or near the absorber. The subsequent slow rise in poloidal flux, at the same time as the slow rise in the toroidal plasma current, starts from a base (just before $t=1 \mathrm{~ms}$ ) that is significantly smaller than the prescribed injector flux of $6 \mathrm{mWb}$, with a total rise in the measured flux of more than $13 \mathrm{mWb}$. The coincident post-formation slow rises in $I_{p}$ and the measured flux suggest a common cause or mechanism, a point that will be explored more carefully below. Note, this slow post-formation buildup in toroidal plasma current and poloidal flux has not been previously observed in ST CHI discharges.

Regarding the amount of toroidal plasma current generated in CHI plasmas in this operating regime, consider the maximum $I_{p} / I_{\mathrm{TF}}$ acheived in $307 \mathrm{HIT}-\mathrm{II}$ CHI discharges, shown in Fig. 6a. Empirically, $I_{p} / I_{\mathrm{TF}}$ can be up to 0.6 at or near the threshold value of $\lambda_{\mathrm{INJ}} d \approx 1 / 3$, with the highest measured $I_{p} / I_{\mathrm{TF}}$ increasing with increasing $\lambda_{\mathrm{INJ}} d$. This is intuitive, as $I_{p} / I_{\mathrm{TF}}$ is proportional to $\lambda_{\text {TOK }}$, and in these CHI discharges $\lambda_{\text {TOK }} \leq \lambda_{\text {INJ }}$, with near-equality for the highest-performance discharges. This result is consistent with the paradigm of the ST plasma generated and sustained by the injector through Taylor relaxation ${ }^{(3)}$.

The measured edge ion temperature, which stays consistently high or even begins dropping while the plasma current is rising and the injector voltage is constant, is also consistent with the picture of the ST plasma sustained by the injector. As the closed flux and current build up in the main discharge, the inverse scale length $\lambda_{\text {TOK }}$ increases, which intuitively should decrease the drive from the injector. A significant decrease in the injector drive would also be a decrease in the edge magnetic relaxation activity, which in turn reduces the source of heat energy for the ions. A strong reduction to the ion heat source in the highly collisional edge should be reflected as a drop in the steady-state ion temperature, similar to the $T_{i}$ drop shown in Fig. 5. Of course, the key assumption here is that the dominant ion heat source is magnetic relaxation activity.

A straightforward explanation for the observed flux amplification in these recent $\mathrm{CHI}$ discharges but not in preceding HIT-II CHI studies is suggested by Figs. 6b and 7 . The product $\lambda_{\text {INJ }} d$ is effectively a measure of the magnetic field pitch in the injector, and will increase if either the toroidal field is decreased or the poloidal injector flux is increased. The significance of $\lambda_{\mathrm{INJ}} d$ is apparent in light of the study described in Ref. ${ }^{(12)}$, in which the rate of field-line reconnection was experimentally demonstrated to be strongly affected by the angle between the reconnecting field lines: antiparallel field lines have the fastest reconnection rate, and decreasing the angle between the field lines strongly decreases the reconnection rate. In the injector region of a $\mathrm{CHI}$ discharge, the nonparallel field lines could be expected to relax or reconnect and form an X-point. Any closed flux formed by this relaxation would be subject to resistive decay, the rates at which the closed current and flux could build up are determined by the relative rates of magnetic relaxation and resistive decay. If the relaxation rate is too small (too large of a toroidal field or too small of an injector flux), then resistive decay would dominate, and current and flux could not build 
up. Previous HIT-II CHI studies have used very high toroidal fields and very low injector fluxes, to minimize the current from the sustainment bank; these studies correspond to values of $\lambda_{\mathrm{INJ}} d<0.3$, below the apparent threshold for current and flux build-up.

\section{Conclusions}

To summarize, a new CHI operating regime has been found in the HIT-II ST device. This regime is characterized by relatively low toroidal field, compared to the poloidal injector flux, and toroidal plasma current that continues to increase after the rapid "bubble-burst" into the confinement region. These CHI discharges consistently have peak plasma currents at or above $300 \mathrm{kA}$, reaching peaks of over $350 \mathrm{kA}$. Internal probing of these discharges shows the "bubble-burst" is associated with the injector flux being pushed into the confinement region, but the subsequent current rise is associated with significant poloidal flux generation and the formation of a closed-current core. Ion Doppler measurements imply that the edge ion temperatures hold steady or even begin dropping as the closed-flux current builds up to its maximum value, for which $\lambda_{\mathrm{TOK}}=\lambda_{\mathrm{INJ}}$. Analysis suggests that access to this $\mathrm{CHI}$ operating regime is related to the field-line geometry in the injector, with significant postbubble-burst current rise correlating with either low $I_{\mathrm{TF}}$ or high injector flux (equivalently, with large field-line pitch across the injector gap from one $\mathrm{CHI}$ electrode to the other).

\section{Acknowledgment}

The authors thank Professor Masayoshi Nagata (Hyogo University, Japan) for the use of the ion Doppler spectrometer used in these experiments, and "Bick" Hooper (Lawrence Livermore National Laboratory, USA) for helpful discussions regarding the IDS data. We also thank George Andexler and John Rogers for their technical assistance in the operation and maintenance of HIT-II, and Rabih AboulHosn for his work on internal probe data. We especially thank the Los Alamos National Laboratory for other equipment used in these studies. This work was supported by the United States Department of Energy.

(Manuscript received Jan. 25, 2005,

revised July 15, 2005)

\section{References}

(1) T. R. Jarboe, M. A. Bohnet, A. T. Mattick, B. A. Nelson, and D. J. Orvis: Phys. Plasmas, Vol.5, p.1807 (1998)

(2) A. J. Redd, B. A. Nelson, T. R. Jarboe, P. Gu, R. Raman, R. J. Smith, and K. J. McCollam: Phys. Plasmas, Vol.9, p.2006 (2002) (Invited Paper for 2001 APS-DPP Meeting)

(3) T. R. Jarboe: Fusion Technology, Vol.15, p.7 (1989)

(4) R. Raman, T. R. Jarboe, B. A. Nelson, W. T. Hamp, V. A. Izzo, R. G. O'Neill, A. J. Redd, P. E. Sieck, and R. J. Smith: Phys. Plasmas, Vol.11, p.2565 (2004)

(5) P. Gu, M. Nagata, K. J. McCollam, T. R. Jarboe, B. A. Nelson, and A. J. Redd: Rev. Scientific Instruments, Vol.75, p.1337 (2004)

(6) B. A. Nelson, T. R. Jarboe, D. J. Orvis, L. A. McCullough, J. Xie, C. Zhang, and L. Zhou: Phys. Rev. Lett., Vol.72, p.3666 (1994)

( 7 ) D. J. Den Hartog, D. J. Craig, G. Fiksel, and J. S. Sarff: Plasma Sources Sci. Tech., Vol.6, p.492 (1997)

( 8 ) T. R. Jarboe, P. Gu, V. A. Izzo, P. D. Jewell, K. J. McCollam, B. A. Nelson, R. Raman, A. J. Redd, P. E. Sieck, R. J. Smith, et al.: Nucl. Fusion, Vol.41, p.679 (2001)

(9) A. J. Redd, W. T. Hamp, V. A. Izzo, T. R. Jarboe, B. A. Nelson, R. G. O'Neill, R. Raman, P. E. Sieck, and R. J. Smith: Bulletin American Phys. Soc., Vol.49, p.304 (2004)

(10) M. Nagata, T. Kanki, N. Fukumoto, and T. Uyama: Phys. Plasmas, Vol.10, p.2932 (2003)

(11) D. J. Orvis: Profile and Stability Studies on the Helicity Injected Tokamak, Ph.D. thesis, University of Washington, Aerospace and Energetics Research, Seattle, Washington (1997)

(12) Y. Ono, A. Morita, and M. Katsurai: Phys. Fluids B, Vol.5, p.3691 (1993)

Aaron J. Redd (Non-member) received his undergraduate

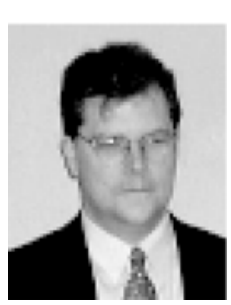
degree in 1993 from the University of Idaho, in the areas of mathematics and physics. His dissertation work on tokamak transport modelling and gyrokinetic stability analysis for pressure-driven kinetic MHD ballooning modes was completed in 1998 at Lehigh University. Presently a Research Associate for the Helicity Injected Torus program at the University of Washington, he has been working on experimental studies of helicity injection current drive in a spherical torus. He is a full member of both the American Physical Society and Sigma Xi.

Will T. Hamp (Non-member) received his undergraduate degree in aerospace engineering from the University of Michigan in 1993 . He worked as a consultant in the automotive industry on a variety of projects, eventually specializing in the dynamic simulation of passenger seats for crashworthiness. This work brought him to Seattle where he continued passenger seat crashworthiness studies at The Boeing Company. He left Boeing in 2000 to pursue advanced degrees, and is currently pursuing his doctorate in plasma physics, specializing in Thomson scattering and other plasma diagnostics.

Thomas R. Jarboe (Non-member) received his undergrad-

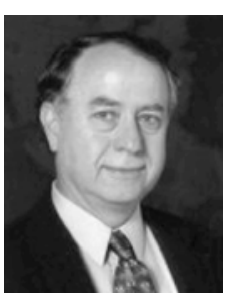
uate degree in Engineering Physics from the University of Illinois in 1967 . He worked a short time for Olin Matheson in East Alton, Illinois and then pursued a doctoral degree at the University of California, Berkeley. In 1974, he received his Ph.D. in plasma physics. He then joined the controlled fusion research division at Los Alamos National Laboratory. He served as group leader from 1983 to 1989 where he studied a new magnetic fusion confinement device called the spheromak. He spent one year beginning in 1985 doing controlled fusion research at Culham Laboratory in England. $\mathrm{He}$ came to the University of Washington in 1989 as Professor of Nuclear Engineering and joined the Department of Aeronautics and Astronautics in 1992. He is a fellow of the American Physical Society. 
Brian A. Nelson (Non-member) completed his plasma

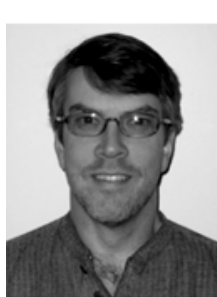
physics dissertation work at the University of Wisconsin, Madison, and then came to the University of Washington in 1987. He has worked on the High-Beta-Q Machine and the Helicity Injected Torus project, and is co-PI for the ZaP experiment, a flow-stabilized Zpinch. He is a full member of the American Physical Society.

R. Griff O'Neill (Non-member) is a physics Ph.D. student

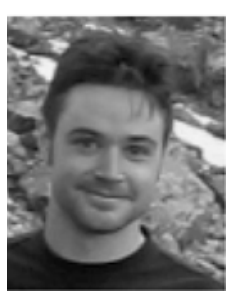
at the University of Washington. His interest is in experimental plasma physics and controlled fusion, and he is currently involved with the Helicity Injected Torus program. His research work has focussed on plasma diagnostics and data analysis, including far-infrared interferometry and ion doppler spectroscopy.
Roger J. Smith (Non-member) completed his disserta-

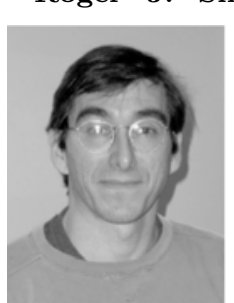
tion work at the University of Washington in physics characterizing the magnetic equilibria of the Coaxial Slow Source FRC device in 1989. He subsequently invented the Transient Internal Probe technique (TIP) to access the core of much hotter plasmas. He then worked for several years on a microwave and interfeometry diagnostic systems at the Joint $\mathrm{Eu}-$ ropean Torus. Since 1998, he has been the lead diagnostician for the Helicity Injected Torus program at the University of Washington. He is a full member of the American Physical Society.

$\begin{array}{lll}\diamond & \diamond & \diamond \\ \diamond & \diamond\end{array}$

